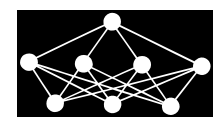

\title{
AUTOMATIC CLASSIFICATION OF AGRICULTURAL GRAINS: COMPARISON OF NEURAL NETWORKS
}

\author{
A. Kayabasi, A. Toktas, K. Sabanci*,E. Yigit*
}

\begin{abstract}
In this study, applications of well-known neural networks such as artificial neural network (ANN), adaptive neuro-fuzzy inference system (ANFIS) and support vector machine (SVM) for wheat grain classification into three species are comparatively presented. The species of wheat grains which are Kama ( $\sharp 70)$, Rosa $(\sharp 70)$ and Canadian $(\sharp 70)$ are designated as outputs of neural network models. The classification is carried out through data of wheat grains $(\sharp 210)$ acquired using $\mathrm{X}$-ray technique. The data set includes seven grain's geometric parameters: Area, perimeter, compactness, length, width, asymmetry coefficient and groove length. The neural networks input with the geometric parameters are trained through 189 wheat grain data and their accuracies are tested via 21 data. The performance of neural network models is compared to each other with regard to their accuracy, efficiency and convenience. For testing data, the ANN, ANFIS and SVM models numerically calculate the outputs with mean absolute error (MAE) of 0.014, 0.018 and 0.135 , and classify the grains with accuracy of $100 \%, 100 \%$ and $95.23 \%$, respectively. Furthermore, data of 210 grains is synthetically increased to 3210 in order to investigate the proposed models under big data. It is seen that the models are more successful if the size of data is increased, as well. These results point out that the neural networks can be successfully applied to classification of agricultural grains whether they are properly modelled and trained.
\end{abstract}

Key words: classification, agricultural grains, wheat grains, neural networks, artificial neural network (ANN), adaptive neuro-fuzzy inference system (ANFIS), support vector machine (SVM)

Received: January 31, 2017

DOI: $10.14311 /$ NNW.2018.28.013

Revised and accepted: April 24, 2018

\section{Introduction}

The agricultural grains such as wheat, rice, barley and lentil which should be assorted into various species and geometric features can be manually or automatically

\footnotetext{
*Ahmet Kayabasi - Corresponding author; Abdurrahim Toktas; Kadir Sabanci; Enes Yigit; Department of Electrical and Electronics Engineering,Karamanoglu Mehmetbey University 70100, Karaman, Turkey, E-mail: ahmetkayabasi@kmu.edu.tr; atoktas@kmu.edu.tr, kadirsabanci@kmu.edu.tr, enesyigit@kmu.edu.tr
} 
appreciated. In manually, the grains are classified based on an expert view. However the decision of the expert can be inaccurate for classifying the species, since the grains are very small and similar to each other. Moreover, employing experts might yield an increase in the cost of product. In recent years, automatic classification of the agricultural grains with the help of computer vision $[2,5,18,19,22]$ has gained importance with respect to the cost and quality. To achieve automatic classification, well-known neural networks [4,9,12,14-17] such as artificial neural network (ANN) [7], adaptive neuro-fuzzy inference system (ANFIS) [11] and support vector machine (SVM) [21] can be integrated with computer vision.

Several studies regarding the classification of agricultural products using ANN, ANFIS and SVM have been proposed in the literature. The efficiencies of the cotton seeds were determined in [10] using classifier ANN. In [15], ANN and ANFIS were utilized to classify rice grains into five species regarding the morphologic features. ANN was modelled for classification of coffee grains according to their mineral content in [13]. An ANN model was designed in [4] for classification of green coffee grains into four group. As the literature is reviewed, the neural networks were utilized for classifying varieties of agricultural grains. The proposed approaches highly depend upon the features of grains taken into account and they varied in classification accuracy. Since there has not been a comprehensive comparative study for these well-known neural networks for classification issues, it is still an important research area which neural network is most effective for classification of grains.

In this study, the neural networks are modelled to classify wheat grains into three different species. ANN, ANFIS and SVM are considered for the classification, since they are the most used neural networks. In this context, data set includes seven geometric parameters of 210 wheat grains given in the literature [2] are employed. These geometric parameters are area, perimeter, compactness, length, width, asymmetry coefficient and groove length for each grain. From the data set, 189 and 21 wheat grains which are according to $90 \%$ and $10 \%$ of wheat grain data are uniformly selected to train and test the accuracy of the models, respectively. Therefore, these neural networks are compared to each other in terms of accuracy, efficiency and convenience. The ANN, ANFIS and SVM models respectively compute the output with mean absolute error (MAE) of $0.007,0.016$ and 0.001 and all models successfully classify the training wheat grains with $100 \%$. On the other hand, they calculate with the MAE of $0.014,0.018$ and 0.135 and accurately sort the testing wheat grains with $100 \%, 100 \%$ and $95.23 \%$, respectively. In order to verify the performance of the models under big data, data of 210 what grains is increased to 3210 by using a clustering technique. The models are trained with data of $2889(90 \%)$ and tested with $321(10 \%)$. MAEs of testing are $0.002,0.020$ and 0.042 for ANN, ANFIS and SVM, respectively. Therefore, the proposed models stably and accurately classify wheat grains under big data, as well.

The presented study is conducted as follows: Section 2 describes acquisition and features of grain data. In Section 3, the principles of neural networks are well addressed; and procedures for modelling and training are outlined. Testing and comparison of the neural network models are explained in Section 4. Eventually, Section 5 presents a conclusion. 


\section{Data Set}

The classification is carried out through a data set reported elsewhere [2] containing three species of wheat grains: Kama, Rosa and Canadian each of which species has 70 grains. The geometric features of the grains were acquired using soft X-ray technique due to being non-destructive and having high quality visualization. The images were taken on $13 \times 18 \mathrm{~cm}$ X-ray Kodak plates. The X-ray photograms were scanned using the Epson Perfection V700 table photo-scanner with a built-in transparency adapter, 600 dpi resolution and 8 bit gray scale levels. For constituting data set, seven geometric parameters of grains: Area (Are), perimeter (Per), compactness $\left(\mathrm{Com}=4 \pi\right.$ Are $\left./ \mathrm{Per}^{2}\right)$, length (Len), width (Wid), asymmetry coefficient (Asc) and groove length $(\mathrm{Grl})$ were extracted from the number of 210 grains. In Fig. 1, 3D scattering of the grains are illustrated to show how Kama, Rosa and Canadian wheat grains discriminate among each other in accordance with the geometric parameters. Len, Wid and Grl (see Fig. 1a); Are, Com and Asc (see Fig. 1b); Are, Per and Len (see Fig. 1c); and Per, Len and Wid (see Fig. 1d) are considered for examining their behavior. It is observed that Kama, Rosa and Canadian wheat grains distinctly cluster with respect to the geometric parameters (see Fig. 1a, 1c and 1d). Although the grains in Fig. 1b also tend to cluster, some of them remain mixed. Hence Kama, Rosa and Canadian wheat grains have different geometric parameters so that they can be successfully utilized to classify the wheat grains.

\section{Neural Networks}

Neural network is a computational model that is inspired by working of biological neural system. Neural networks consist of a group of neurons which processes information through interconnection [8]. The neural networks of ANN, ANFIS and SVM are a powerful method being capable of accurately predicting, estimating or classifying. The neural networks are classification, regression and prediction tools that use machine learning theory to maximize predictive accuracy while automatically avoiding over-fit to the data $[1,9,14-16]$. The modelling and training of the neural networks for classification of wheat grains is described below.

\subsection{Modelling}

A graphical user interface (GUI) illustrated in Fig. 2 is designed in platform of MATLAB $\AA$. Behind the GUI, the neural networks models previously modelled and properly trained operate, and thus a user can easily classify wheat grain species. Block diagrams of the neural network models for the classification are given in Fig. 3c and set parameters that used in the models are tabulated in Tab. I. The geometric parameters of Are, Per, Com, Len, Wid, Asc, and Grl are considered as inputs of the ANN, ANFIS and SVM models. Kama, Rosa and Canadian wheat species are considered as output parameters.

ANN consists of neurons organized into different layers. These neurons containing non-linear types of functions are mutually connected by synaptic weights [7]. In order to classify the wheat grains into Kama, Rosa or Canadian according to the geometric parameters, ANN model based on multilayer perceptron is designed 


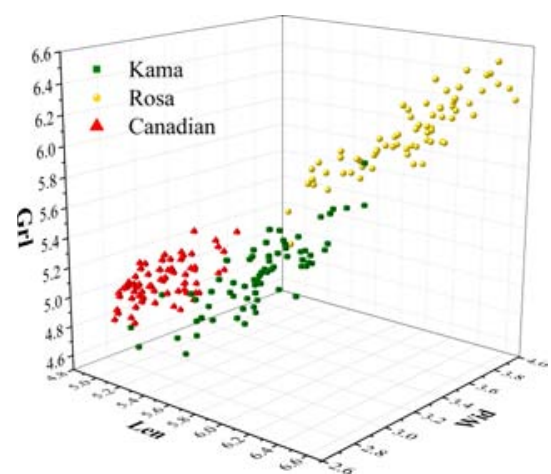

(a)

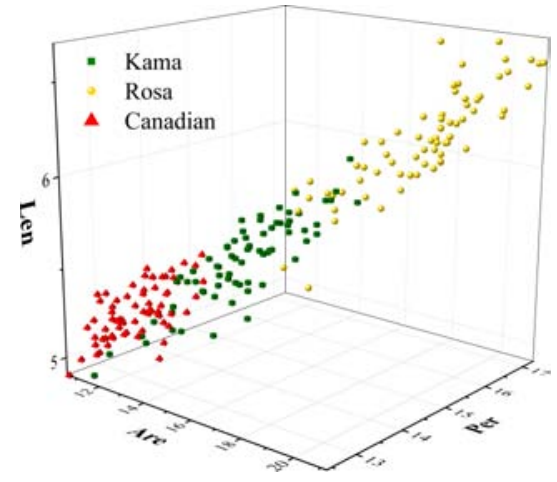

(c)

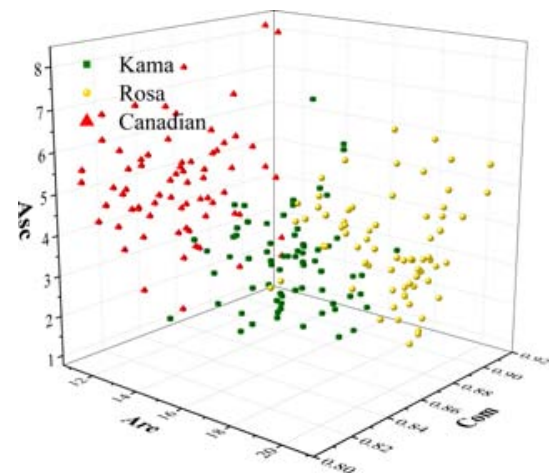

(b)

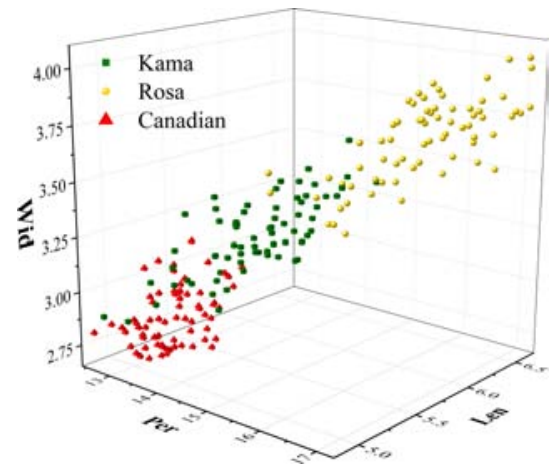

(d)

Fig. $13 \mathrm{D}$ scattering of 210 wheat grains according to geometric parameters of (a) Len, Wid and Grl; (b) Are, Com and Asc; (c) Are, Per and Len; (d) Per, Len and Wid.

as shown in Fig. 3a along with the set parameters given in Tab. I. The model is constructed with three layers: input layer, hidden layer with 5 neurons and output layer with 1 neuron. The neuron number of input layer equals to the number of input parameters. A function given in Eq. 1 processes in each neuron.

$$
y_{j}=f\left(\sum w_{j i} x_{i}\right)
$$

here, $i$ and $j$ are respectively indexes for neurons of input and hidden layers. Neurons in the input layer act as buffers for distributing the input signals $x_{i}$ to neurons in the hidden layer. Each neuron $j$ in the hidden layer sums up its input signals $x_{i}$ after weighting them with the strengths of the respective connections $w_{j i}$ from the input layer and computes its output $y_{j}$ as a function $f$ of the sum. During training, these strengths weaken or strengthen to bring closer the output to the target of the network. $f$ can be a simple threshold function such as sigmoid, hyperbolic tangent, tangent sigmoid (2), radial basis, purelin (3) etc [8]. Tangent sigmoid function in both input and hidden layers, purelin function in output layer are used in our model. 
Kayabasi A. et al.: Automatic classification of agricultural grains: comparison of...

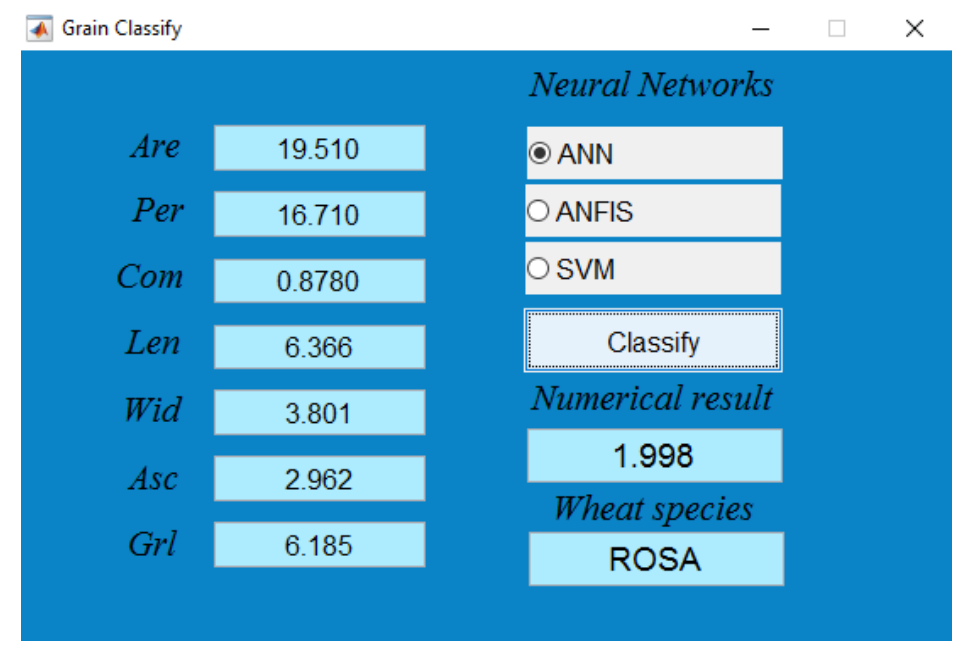

Fig. 2 Graphical user interface for the neural network models.

$$
\begin{gathered}
f(x)=\frac{2}{1+e^{-2 x}}-1 \\
f(x)=x
\end{gathered}
$$

In the contrast to ANN, fuzzy inference system (FIS) has not capability of learning and updating itself according to environment conditions. ANFIS combines the learning property of ANN with expert knowledge of FIS. Thanks to the ANN, the ability of learning is provided by optimizing the linguistic expressions which are the basis of the FIS [11]. ANFIS consists of different layers, which have nodes, each performs a specific task. In general, ANFIS is composed of 5 layers that include 1 input, 3 hidden and 1 output layers. Hidden layers consist of two membership functions (MFs) of input and output layers and one fuzzy logic rule layer. In classification of wheat grains, an ANFIS model based on Sugeno type FIS [20] with seven rules is designed as depicted in Fig. 3b. For input and output MFs, Gaussian (4) and linear functions (3) are respectively utilized. The nodes in input MF layer fuzzificate the numerical inputs of the networks. In this layer, membership degree of the MF is determined for each numerical input of the nodes. Fuzzy inputs of each node are then processed according to defined rules using logical operators in the rules layer. The fuzzy outputs of rule layer are then de-fuzzificated in the output MF layer to obtain numerical results. Finally, real result is computed by summing these numerical values in the output layer.

$$
f(x)=e^{-\frac{(x-c)^{2}}{2 \sigma^{2}}},
$$

where $c$ and $\sigma$ are respectively the center and width of Gaussian curve.

SVM is a recent powerful neuro machine algorithm based on statistical learning theory. Mathematical algorithms of SVM are firstly designed to classify two linear 


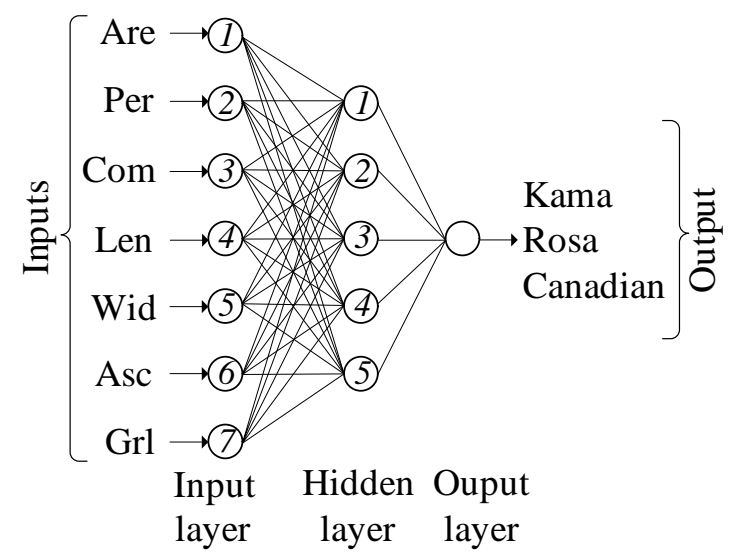

(a) ANN

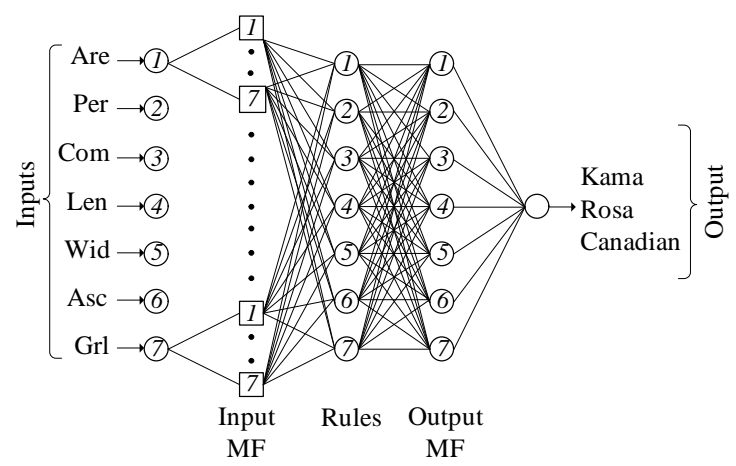

(b) ANFIS

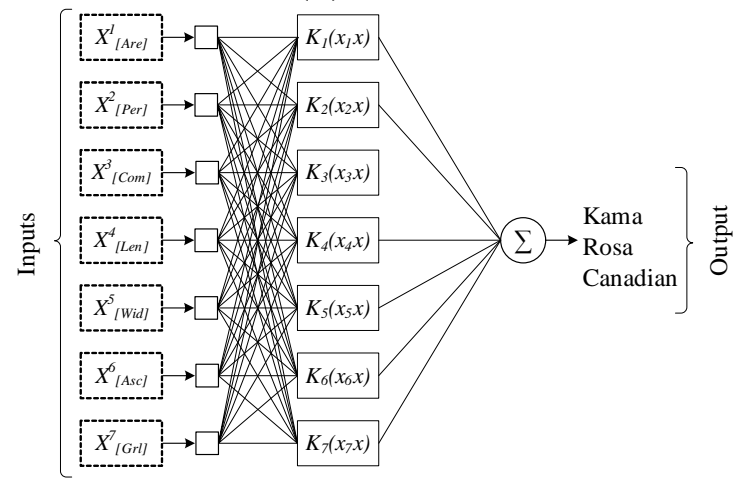

(c) SVM

Fig. 3 Models of the neural networks: (a) ANN; (b) ANFIS; (c) SVM.

data problems, and then have been generalized for classifying multi-class nonlinear data and regression processes. Therefore, SVM can separate two data groups by $\mathrm{N}$-dimension optimum hyperplane by using the structural risk minimization $[3$, 
Kayabasi A. et al.: Automatic classification of agricultural grains: comparison of...

\begin{tabular}{llc}
\hline Models & Parameters & Set type/value \\
\hline \multirow{4}{*}{ ANN } & Epochs & 250 \\
& Minimum gradient descent & $10^{-10}$ \\
& Momentum parameter $(\mu)$ & 0.0001 \\
& $\mu$ increment & 0.1 \\
& $\mu$ decrement & 4 \\
\hline \multirow{4}{*}{ ANFIS } & Epochs & 150 \\
& Range of influence & 0.5 \\
& Squash factor & 1.125 \\
& Accept ratio & 0.5 \\
& Reject ratio & 0.15 \\
\hline \multirow{3}{*}{ SVM } & Kernel function & Gaussian \\
& Kernel function coefficient $(\sigma)$ & 0.05 \\
& Penalty weight $(C)$ & 1000000 \\
& Slack variables (epsilon- $\xi)$ & 0.001 \\
\hline
\end{tabular}

Tab. I Set parameters of the neural networks.

21]. The key point in SVM is linear discriminant function called hyperplane (or margin) reflecting the characteristics of data-set. SVM effectively establishes an optimum hyperplane having equidistant from both of the data in a high or infinite dimensional space. It thus easily finds a solution for linearly separable problems. In order to solve nonlinear problems, data is mapped to higher dimensional space by using kernel function, and then linear classifier is used in the higher dimensional space [3,21]. SVM network generally has two feed-forward layers as similar to ANN. SVM modelled in this study for wheat grain classification is illustrated in Fig. 3c. Gaussian Kernel function [21] given in Eq. 5 is utilized in the SVM model

$$
k(x, y)=\exp \left(-\frac{\|x-y\|^{2}}{2 \sigma^{2}}\right) .
$$

\subsection{Training}

A flowchart for training the neural networks is shown in Fig. 4. The total number of 210 wheat grains are uniformly separated by $90 \%$ and $10 \%$ for training and testing process, respectively. Note that the wheat grains used in these process is randomly selected from whole data set in order to represent the entire solution space. Therefore, the neural network models are trained through the data of 189 geometric parameters of wheat grains.

According to the flowchart, the neural network models are trained (optimized) by different algorithms: ANN by Levenberg-Marquardt learning algorithm [6]; ANFIS by hybrid-learning algorithm [11]; SVM by quadratic programming [3]. The flowchart of training process mainly consists of five steps. Training data of 189 wheat grains is loaded in the first step. In the second step, desired model is selected as ANN, ANFIS or SVM with specific set parameters and then the selected 
model is being trained through the loaded data. In third step, training results are checked according to the following MAE,

$$
\mathrm{MAE}=\frac{\sum(\text { Target }- \text { Output })}{\text { Number of grains }}
$$

where target is " 1 ", " 2 " or " 3 " which respectively correspond to Kama, Rosa and Canadian wheat grain species. Output is the numerical result yielded by the neural network for the same grain. If the MAE is less than objective value of 0.2 , the successful trained model is saved. Otherwise, the training step repeats until achieving the objective value. The training results are revealed at the last step.

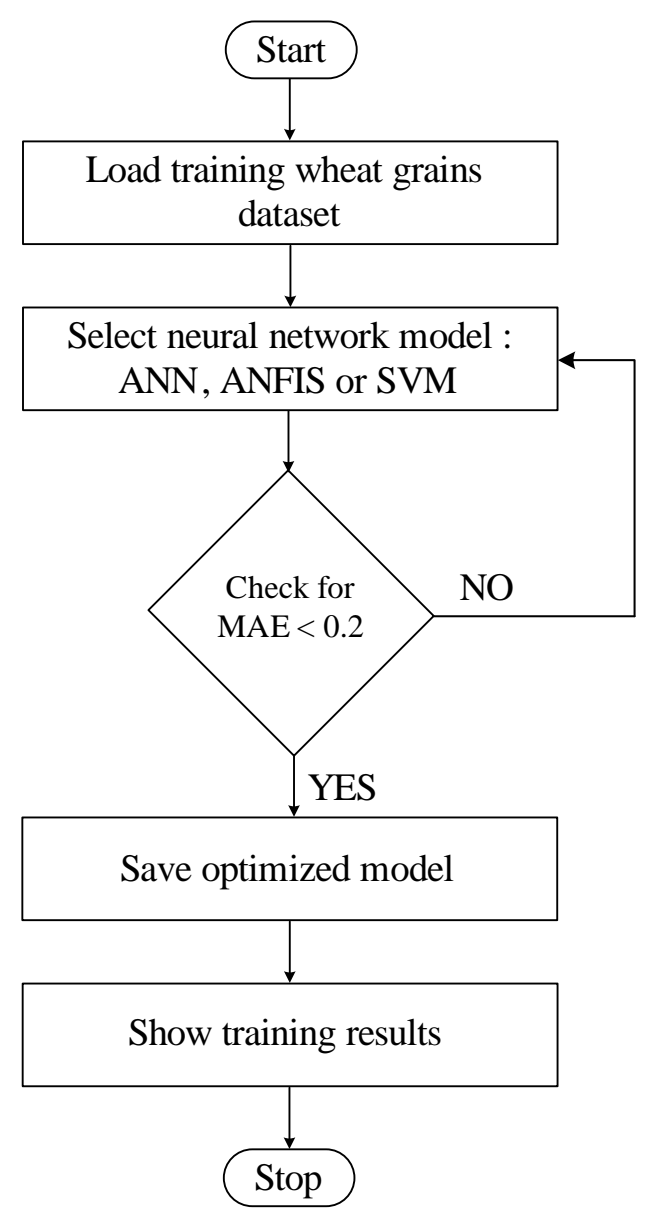

Fig. 4 Flowchart of training process of neural network models.

Numerical and classification results of the neural networks are comparatively illustrated in Fig. 5. The neural networks classify wheat grains into Kama, Rosa or Canadian if numerical results are in the range of $0.51-1.50,1.51-2.50$ and 2.513.54, respectively. From Fig. 5a, the neural networks of ANN, ANFIS and SVM 
are properly trained with the MAE of $0.007,0.016$ and 0.001 , respectively. As seen from Fig. 5b, the ANN, ANFIS and SVM models determine the wheat species with accuracy of $100 \%$. Therefore, all models accurately classify entire 189 wheat species for training.

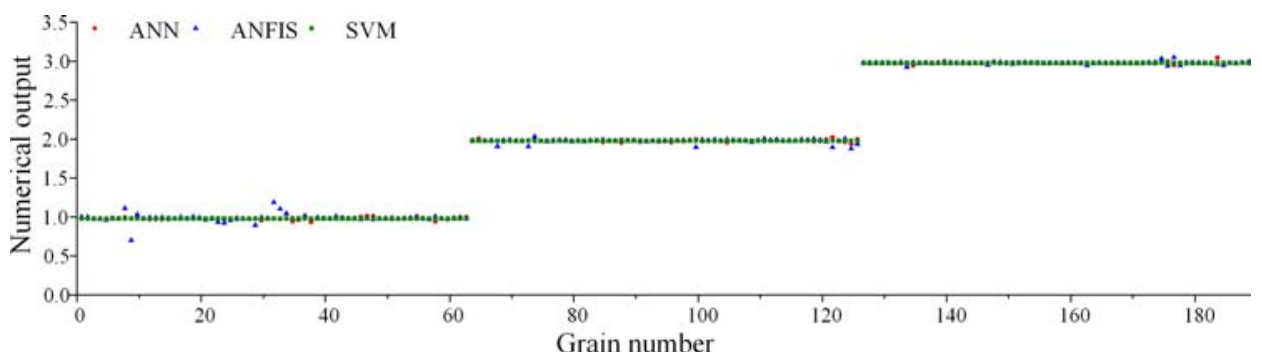

(a) Numerical

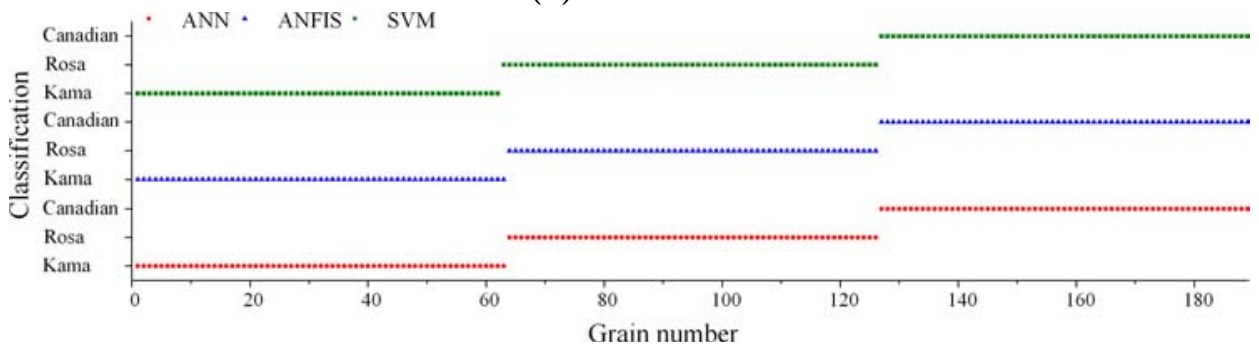

(b) Classification

Fig. 5 Comparative training results of the neural network models.

\section{Test and Comparison of Neural Networks}

In order to test the accuracy of the neural network models, data of 21 wheat grains is selected randomly from the total of 210 wheat grains. It is worth nothing that the wheat grains selected for testing were not used in the training process. To analysis the testing process in detail, the geometric parameters and the neural networks' results are listed in Tab. II. The ANN, ANFIS and SVM models classify the wheat species with accuracy of $100 \%$ (MAE: 0.014), $100 \%$ (MAE: 0.018) and $95.23 \%$ (MAE: 0.135 ), respectively. The ANN and ANFIS models hence correctly determine the whole 21 wheat species; on the other hand, the SVM model accurately classify those of 20 wheat species for testing. The neural networks of ANN, ANFIS and SVM modelled in this study are successfully implemented to classify the wheat grains. From the testing results presented in Fig. 6, although the MAE of the ANN and ANFIS models are 0.014 and 0.018 (see Fig. 6a), they accurately classify the whole of grains into Kama, Rosa or Canadian (see Fig. 6b). Whereas the SVM model correctly determines 20 species of 21 wheat grains with the MAE of 0.135 . In the perspective of design and optimization models of the neural networks, the implementation of ANN is simpler and easier than the others for this 
Neural Network World 3/2018, 213-224

\begin{tabular}{|c|c|c|c|c|c|c|c|c|c|c|c|c|c|c|c|c|c|}
\hline \multirow{3}{*}{$\begin{array}{c}\text { Grain } \\
\sharp\end{array}$} & \multicolumn{6}{|c|}{ Geometric parameters } & \multirow{3}{*}{ Grl } & \multicolumn{10}{|c|}{ Neural network results } \\
\hline & \multirow[t]{2}{*}{ Are } & \multirow[t]{2}{*}{ Per } & \multirow[t]{2}{*}{ Com } & \multirow[t]{2}{*}{ Len } & \multirow[t]{2}{*}{ Wid } & \multirow[t]{2}{*}{ Asc } & & \multirow[t]{2}{*}{ Target } & \multicolumn{3}{|c|}{ Numerical output } & \multicolumn{3}{|c|}{ Absolute Error } & \multicolumn{3}{|c|}{ Classification } \\
\hline & & & & & & & & & ANN & ANFIS & SVM & ANN & ANFIS & SVM & ANN & & SVM \\
\hline 1 & 14.690 & 14.490 & 0.880 & 5.563 & 3.259 & 3.586 & 5.219 & 1 & 0.987 & 0.996 & 1.134 & 0.013 & 0.004 & 0.134 & Kama & Kama & Kama \\
\hline 2 & 15.010 & 14.760 & 0.866 & 5.789 & 3.245 & 1.791 & 5.001 & 1 & 1.001 & 1.048 & 0.991 & 0.001 & 0.048 & 0.009 & Kama & Kama & Kama \\
\hline 3 & 13.990 & 13.830 & 0.918 & 5.119 & 3.383 & 5.234 & 4.781 & 1 & 1.023 & 1.009 & 2.108 & 0.023 & 0.009 & 1.108 & Kama & Kama & Rosa \\
\hline 4 & 15.110 & 14.540 & 0.899 & 5.579 & 3.462 & 3.128 & 5.180 & 1 & 1.013 & 0.953 & 1.166 & 0.013 & 0.047 & 0.166 & Kama & Kama & Kama \\
\hline 5 & 14.790 & 14.520 & 0.882 & 5.545 & 3.291 & 2.704 & 5.111 & 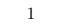 & 1.005 & 1.006 & 0.957 & & & & Kama & & Kama \\
\hline 6 & 15.880 & 14.900 & 0.899 & 5.618 & 3.507 & 0.765 & 5.091 & 1 & 1.001 & 1.02 & 1.248 & 0.001 & & & Kan & Kama & Kama \\
\hline 7 & 14.090 & 14.410 & 0.853 & 5.717 & 3.186 & 3.920 & 5.299 & 1 & 0.996 & 1.003 & 1.197 & 0.004 & 0.003 & 0.197 & Kama & Kama & Kama \\
\hline 8 & 19.510 & 16.710 & 0.878 & 6.366 & 3.801 & 2.962 & 6.185 & 2 & 1.998 & 2.003 & 1.990 & 0.002 & 0.003 & 0.010 & Rosa & Rosa & Rosa \\
\hline 9 & 18.890 & 16.230 & 0.901 & 6.227 & 3.769 & 3.639 & 5.966 & 2 & 2.204 & 2.013 & 1.995 & 0.204 & 0.013 & 0.005 & Rosa & Rosa & Rosa \\
\hline 10 & 18.940 & 16.320 & 0.894 & 6.144 & 3.825 & 2.908 & 5.949 & 2 & 1.986 & 2.007 & 2.002 & 0.014 & & 0.002 & Rosa & Rosa & Rosa \\
\hline 11 & 17.550 & 15.660 & 0.899 & 5.791 & 3.690 & & 5.6 & 2 & & & & & & & & & Rosa \\
\hline 12 & 19.140 & 16.610 & 0.872 & 6.259 & 3.737 & & & & & & & & & & & & Rosa \\
\hline 13 & 18.720 & 16.340 & 0.881 & 6.219 & 3.684 & 2.188 & 6.097 & 2 & 2.000 & 2.008 & 2.018 & 0.000 & 08 & & osa & Ro & Rosa \\
\hline 14 & 17.990 & 15.860 & 0.899 & 5.890 & 3.694 & 2.068 & 5.837 & 2 & 1.999 & 2.120 & 1.961 & 0.001 & 0.120 & 0.039 & Rosa & Rosa & Rosa \\
\hline 15 & 10.740 & 12.730 & 0.833 & 5.145 & 2.642 & 4.702 & 4.963 & 3 & & 3.001 & 3.008 & & & 0.008 & Canadian & Canadian & Canadian \\
\hline 16 & 11.180 & 12.720 & 0.868 & 5.009 & 2.810 & 4.051 & 4.828 & 3 & 3.003 & 3.002 & 2.875 & 0.003 & 0.002 & 0.125 & Canadian & Canadian & Canadian \\
\hline 17 & 12.190 & 13.360 & 0.858 & 5.240 & 2.909 & 4.857 & 5.158 & 3 & 2.998 & 3.003 & 2.975 & 0.002 & 0.003 & 0.025 & Canadian & Canadian & Canadian \\
\hline 18 & 10.590 & 12.410 & 0.865 & 4.89 & 2.787 & 4.975 & $4.7 ?$ & 3 & 3.0 & 2.9 & 2.6 & 0.0 & & 0.3 & Canadian & anadian & anadian \\
\hline 19 & 11.550 & 13.100 & 0.846 & 5.167 & 2.845 & 6.715 & 4.9 & 3 & & 2.9 & 2.9 & & & & Cana & Canadian & Canadian \\
\hline 20 & 12.020 & 13.330 & 0.850 & 5.350 & 2.810 & 4.271 & 5.31 & 3 & & 3.008 & 2.9 & 0.002 & & 0.054 & Canadian & Canadian & Canadian \\
\hline \multirow[t]{2}{*}{21} & 10.820 & 12.830 & 0.826 & 5.180 & 2.630 & 4.853 & 5.089 & 3 & 2.998 & 3.002 & 3.040 & 0.002 & 0.002 & 0.040 & Canadian & Canadian & Canadian \\
\hline & & & & & & & & & & & MAE & 0.014 & & 0.13 & & & \\
\hline
\end{tabular}

Tab. II Geometric parameters of wheat grains for testing and the neural networks' results.

task. Computational time for the three models is almost the same for testing process. Note that these results are obtained in the condition of the proposed model and set parameters. The results might be improved with more proper models and setting.

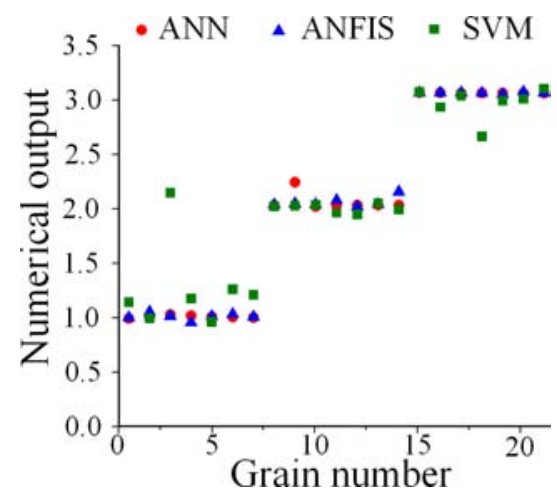

(a) Numerical

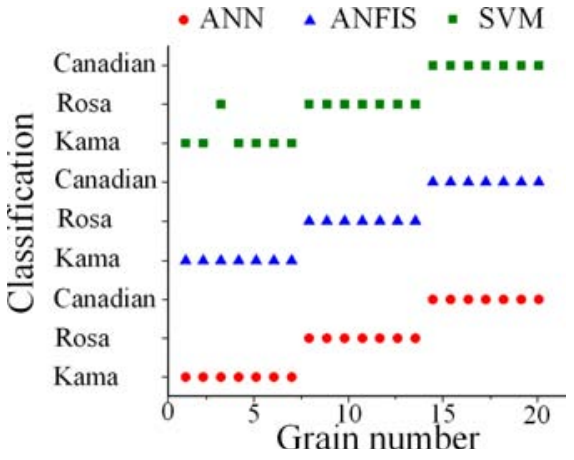

(b) Classification

Fig. 6 Comparative testing results of the neural network models.

One of the most important factor in process of neural networks is the size of data. In the training process, the neural network should be trained with sufficient number of data (input pattern) reflecting the characteristic of the problem for reaching better performance. Therefore, data sets representing the solution space of problem should be utilized as training dataset for investigating the performance of the neural networks. In order to further validate the performance of the neural networks under big data, data of 210 wheat grains is synthetically increased to 3210 by reproducing the real data using a method based on Fuzzy $\mathrm{C}-$ means clustering [23] technique. This is also uniformly separated by $90 \%$ and $10 \%$, as taken in 
standard training and testing processes. Data of 2889 is thus used for training and then the remaining 321 is utilized for evaluating the performance of the models under big data. The achieved the MAE for testing are 0.002, 0.020 and 0.042 for ANN, ANFIS and SVM; and they classifying the grains with the accuracy of $100.00 \%, 99.68 \%$ and $99.37 \%$, respectively. The results show that when the size of data is increased, the accuracy also rises correspondingly. Therefore, proposed neural network models are successful and stable under big data, as well. As a result, the neural networks can be successfully exploited for automatic classification of agricultural grains. It should be noticed that computer systems in which neural networks are running should be fast enough for automatic classification by means of realized mechanical construction with computer vision.

\section{Conclusions}

In this paper, a comparison of different neural networks is successfully carried out for classification of wheat grains into three species of Kama, Rosa or Canadian. The applications of ANN, ANFIS and SVM are conducted through 210 wheat grain data of which geometric parameters acquired using X-ray technique. The training and testing of the neural network models are accomplished by using data of 189 and 21 wheat grains, respectively. The neural networks are compared in terms of classification performance and design procedure. The ANN, ANFIS and SVM models numerically compute the outputs with the MAE of $0.014,0.018$ and 0.135 , and accurately classify the grains with $100 \%, 100 \%$ and $95.23 \%$, respectively, for testing the wheat grains. To examine the performance of the models under big data, data of 210 wheat grains is synthetically augmented to 3210 grains. It shows that the proposed neural network models are more successful and stable under big data, as well. Moreover, it can be said that the modelling of ANN is easier and more flexible than ANFIS and SVM in design and optimization procedures for classification. Consequently, the proposed neural networks can be successfully utilized in automatic classification of agricultural grains if they are properly modelled and integrated with a fast enough realized system.

\section{References}

[1] AKBARIMAJD A., JONBAN M.S., NOOSHYAR M., DAVARI M. Neural network based identification of trichoderma species. Neural Network World. 2016, 26(2), pp. 155-173, doi: 10.14311/NNW.2016.26.009.

[2] ChARYTANOWICZ M., NIEWCZAS J., KULCZYCKI P., KOWALSKI P.A., ŁUKASIK S., ŻAK S. Complete gradient clustering algorithm for features analysis of $x$-ray images. Information Technologies in Biomedicine. 2010, pp. 15-24, Springer, Berlin. Heidelberg, doi: 10.1007/978-3-642-13105-9_2.

[3] CRISTIANINI N., SHAWE-TAYLOR J. An introduction to support vector machines and other kernel-based learning methods. Cambridge: Cambridge University Press., 2000, doi: 10. 1017/CB09780511801389.

[4] DE OliveirA E.M., LEME D.S., BARBOSA B.H.G., RODARTE M.P., PEREIRA R.G.F.A. A computer vision system for coffee beans classification based on computational intelligence techniques. Journal of Food Engineering, 2016, 171, pp. 22-27, doi: 10.1016/j. jfoodeng.2015.10.009. 


\section{Neural Network World 3/2018, 213-224}

[5] GUEVARA-HERNANDEZ F., GOMEZ-GIL J. A machine vision system for classification of wheat and barley grain kernels. Spanish Journal of Agricultural Research, 2011, 9(3), pp. 672-680, doi: $10.5424 / \mathrm{sj}$ ar/20110903-140-10.

[6] HAGAN M.T., MENHAJ M.B. Training feedforward networks with the marquardt algorithm. IEEE Transactions on Neural Networks, 1994, 5(6), pp. 989-993, doi: 10.1109/72. 329697.

[7] HAYKIN S. Neural networks: A comprehensive foundation, Macmillan College Publishing Company, New York, A.B.D, 1994.

[8] HAYKIN S. Neural networks and learning machines (Vol. 3). Upper Saddle River, NJ, USA: Pearson, 2009.

[9] HU B.G., GOSINE R.G., CAO L.X., DE SILVA C.W. Application of a fuzzy classification technique in computer grading of fish products. IEEE Transactions on Fuzzy Systems, 1998, 6(1), pp. 144-152, doi: 10.1109/91.660814.

[10] JAMUNA K.S., KARPAGAVALLI S., REVATHI P., GOKILAVANI S., MADHIYA E. Classification of seed cotton yield based on the growth stages of cotton crop using machine learning techniques. In: Proceedings of the 2010 International Conference on Advances in Computer Engineering, Bangalore, Karnataka, India, 2010, pp. 312-315.

[11] JANG J.S.R., ANFIS: Adaptive-network-based fuzzy inference system. IEEE Transactions on Systems, 1993, 23(3), pp. 665-685, doi: 10.1109/21.256541.

[12] MOLLAZADE K., OMID M., AREFI A. Comparing data mining classifiers for grading raisins based on visual features. Computers and Electronics in Agriculture, 2012, 84, pp. 124-131, doi: 10.1016/j. compag. 2012.03.004

[13] MUÑIZ-VALENCIA R., JURADO J.M., CEBAllOS-MAGAÑA S.G., AlCÁZAR Á. HERNÁNDEZ-DÍAZ J. Characterization of Mexican coffee according to mineral contents by means of multilayer perceptrons artificial neural networks. Journal of Food Composition and Analysis, 2014, 34(1), pp. 7-11, doi: 10.1016/j.jfca.2014.02.003.

[14] OLGUN M., ONARCAN A.O., ÖZKAN K., IŞIK Ş., SEZER O., ÖZGIŞI K., AYTERA N.G., BUDAK BAŞÇIFTÇI Z., ARDIÇ M., KOYUNCU O. Wheat grain classification by using dense SIFT features with SVM classifier. Computers and Electronics in Agriculture, 2016, 122, pp. 185-190, doi: 10.1016/j.compag.2016.01.033.

[15] PAZOKI A.R., FAROKHI F., PAZOKI Z. Classification of rice grain varieties using two Artificial Neural Networks (MLP and Neuro-Fuzzy). Journal of Animal 8 Plant Sciences, 2014, 24(1), pp. 336-343.

[16] SABANCI K., KAYABAŞI A., TOKTAŞ A. Computer vision-based method for classification of wheat grains using artificial neural network. Journal of the Science of Food and Agriculture. 2016, 97(8), pp.2588-2593, doi: 10.1002/jsfa.8080.

[17] SABANCI K., AKKAYA M. Classification of Different Wheat Varieties by Using Data Mining Algorithms. International Journal of Intelligent Systems and Applications in Engineering, 2016, 4(2), pp. 40-44, doi: 10.18201/ijisae.62843.

[18] SZCZYPINSKI P.M., ZAPOTOCZNY P. Computer vision algorithm for barley kernel identification, orientation estimation and surface structure assessment. Computers and Electronics in Agriculture, 2012, 87, pp. 32-38, doi: 10.1016/j.compag.2012.05.014.

[19] SZCZYPINSKI P.M., KLEPACZKO A., ZAPOTOCZNY P. Identifying barley varieties by computer vision. Computers and Electronics in Agriculture, 2015, 110, pp. 1-8, doi: 10. 1016/j. compag. 2014.09.016.

[20] TAKAGI T., SUGENO M. Fuzzy identification of systems and its applications to modeling and control. IEEE transactions on systems, man, and cybernetics, 1985, 15, pp. 116-132, doi: 10.1109/TSMC. 1985.6313399.

[21] VAPNIK V.N. Statistical Learning Theory, Wiley, New York, 1998.

[22] ZAPOTOCZNY P. Discrimination of wheat grain varieties using image analysis: morphological features. European Food Research and Technology. 2011, 233, pp. 769-779, doi: 10.1007/s00217-011-1573-y.

[23] ZHOU K., FU C., YANG S. Fuzziness parameter selection in fuzzy C-means: the perspective of cluster validation. Science China Information Sciences. 2014, 57(11), pp. 1-8. 